\title{
Negative phenotypic and genetic associations between copulation duration and longevity in male seed beetles
}

\author{
EA Brown ${ }^{1,3}$, L Gay ${ }^{1}, \mathrm{R}$ Vasudev ${ }^{2}$, T Tregenza ${ }^{1}$, PE Eady ${ }^{2}$ and DJ Hosken ${ }^{1}$ \\ ${ }^{1}$ Centre for Ecology and Conservation, University of Exeter, Cornwall Campus, Tremough, Penryn, UK and ${ }^{2}$ Department of Biological \\ Sciences, University of Lincoln, Lincoln, UK
}

Reproduction can be costly and is predicted to trade-off
against other characters. However, while these trade-offs are
well documented for females, there has been less focus on
aspects of male reproduction. Furthermore, those studies
that have looked at males typically only investigate pheno-
typic associations, with the underlying genetics often
ignored. Here, we report on phenotypic and genetic trade-
offs in male reproductive effort in the seed beetle, Calloso- bruchus maculatus. We find that the duration of a male's first copulation is negatively associated with subsequent male survival, phenotypically and genetically. Our results are consistent with life-history theory and suggest that like females, males trade-off reproductive effort against longevity.

Heredity (2009) 103, 340-345; doi:10.1038/hdy.2009.80; published online 29 July 2009

Keywords: mating costs; Callosobruchus; heritability; genetic correlation

\section{Introduction}

Mating can be costly, with activities like mate searching, courtship or sexual signalling typically very energetically expensive (for example, Bailey et al., 1993; Kotiaho et al., 1998). Furthermore, there are also opportunity costs to these activities, and often, increased predation risk (Tuttle and Ryan, 1981; Burk, 1982; Hosken et al., 1994). In addition to these costs, which are paid whether or not copulation takes place, there are also potential costs that are directly related to the act of copulation itself. For example, male genitalia or toxic seminal fluid can harm females (for example, Chapman et al., 1995; Crudgington and Siva-Jothy, 2000; Blanckenhorn et al., 2002; Wigby and Chapman, 2005). There may also be costs associated with gamete production (Kenagy and Trombulak, 1986; Van Voorhies, 1992) and seminal fluid-or some other aspects of copulation-can lead to downregulation of the immune system (for example, Siva-Jothy et al., 1998; Hodgson and Hosken, 2006; but see Schwarzenbach et al., 2005).

Costs of copulation are reasonably well documented for females (for example, Thornhill and Alcock, 1983; Arnqvist and Rowe, 2005), but there have been fewer investigations of these costs for males (Hunt et al., 2006). Strategic ejaculation (for example, Gage, 1991; Gage and Baker, 1991; Simmons et al., 1993; Martin and Hosken,

Correspondence: Dr DJ Hosken, Centre for Ecology and Conservation, School of Biosciences, University of Exeter, Cornwall Campus, Tremough, Penryn, TR10 9EZ, UK.

E-mail: d.j.hosken@exeter.ac.uk

${ }^{3}$ Current address: Department of Animal \& Plant Science, University of Sheffield, Sheffield, UK

Received 14 January 2009; revised 12 June 2009; accepted 18 June 2009; published online 29 July 2009
2002; reviewed in Wedell et al., 2002) provides indirect evidence that copulation is costly for males, otherwise facultative ejaculate adjustment would not be advantageous. There is also more direct evidence that copulation is costly for males (for example, Clutton-Brock and Langley, 1997; Martin and Hosken, 2004). For example, longevity is negatively associated with copulation number for male Saltella sphondylli (Martin and Hosken, 2004). However, the few available estimates of cost of copulation for males are typically phenotypic, and it is unclear whether there is a genetic basis to trade-offs between aspects of copulation and other male characters. There are theoretical reasons to expect this would be the case (Roff, 2002), and much of the controversy regarding trade-offs between reproductive investment and other characters is probably due to the fact that these associations are often only measured at the phenotypic level (Reznick, 1985; Roff, 2002). Indeed, some authors have gone so far as to say that without genetic estimates, a bona fide trade-off cannot be shown (Rose and Charlesworth, 1981; Reznick, 1985; but see Roff, 2002).

In addition to the paucity of estimates of male mating costs, estimates of heritability of copulatory behaviour are also relatively uncommon (Mühlhäuser et al., 1996). Behaviour typically has a relatively large environmental component (Mousseau and Roff, 1987), and it is also frequently highly stereotypical, especially mating behaviour, further suggesting there may often be little additive-genetic variation for aspects of mating behaviour. However, copulation duration is heritable in several taxa (for example, MacBean and Parson, 1967; Gromko et al., 1991), including seed beetles (Savalli and Fox, 1998) and can be an important male fitness component (Simmons et al., 1996). 
Here, we estimate the cost of a single copulation in the seed beetle, Callosobruchus maculatus. Previous work on this species indicates that mating can be costly for both males (Paukku and Kotiaho, 2005) and females (Tatar et al., 1993; Crudgington and Siva-Jothy, 2000; Eady et al., 2007). Copulating females kick their mates vigorously during the last third of copulation (Qi and Burkholder, 1982; Eady, 1991). This behaviour reduces copulation duration and mitigates to an extent the injuries caused by the spiny male genitalia (Crudgington and Siva-Jothy, 2000; Edvardsson and Tregenza, 2005). However, there has been no investigation of how, or if, variation in copulation duration influences mating costs in male $C$. maculatus. Moreover, previous estimates of male mating costs are only phenotypic (Paukku and Kotiaho, 2005), and these may or may not reflect genetic associations (Lynch and Walsh, 1998). Our study of the costs of copulation is conducted in a quantitative genetic framework that allows us to estimate the genetic correlation between copulation duration and subsequent male longevity, as well as the heritability of several male fitness components, including copulation duration. Our results indicate that longevity is negatively influenced by the duration of a male's first copulation, at both the phenotypic and genetic levels and that there is significant additive-genetic variation in both characters.

\section{Materials and methods}

C. maculatus is a widely distributed pest on stored legumes. Its eggs are attached to beans, and the larvae develop inside the bean. The beetles used in this experiment were derived from a wild-type stock population, originating from Niamey, Niger and maintained in the University of Lincoln at a population size of approximately 500 individuals on ca. 2000 black-eyed beans Vigna unguiculata at $27^{\circ} \mathrm{C}$ (with a $16: 8 \mathrm{~h}$ light/dark photoperiod) for hundreds of generations. Twenty generations before the experiment, the population size was increased to ca. 2500 individuals (on $250 \mathrm{~g}$ of blackeyed beans). Under normal culture conditions, adult beetles do not feed or drink, surviving instead on resources accrued during the larval stage.

Approximately 200 females from the mass culture were allowed to oviposit for $6 \mathrm{~h}$ on ca. 3000 black-eyed beans. At this density, we expect females to lay one egg per bean (Horng, 1997). Before emergence, randomly chosen beans were removed and isolated in 'virgin chambers' (48-well cell culture plates, VWR International Ltd, Lutterworth, UK). If beans contained more than one emerging adult, the beetles were removed as they emerged, and if this occurred simultaneously, only groups of emerged adults of the same sex were retained and used in subsequent (parental) matings. Adults emerging from these beans were subsequently used to generate sib-ships (full-sib/half-sib families) used to estimate genetic parameters (see Statistical analyses below). Briefly, virgin males (sires) and females (dams) (all $24-48 \mathrm{~h}$ old) were placed in individual $40 \mathrm{~mm}$ diameter Petri dishes for $10 \mathrm{~min}$ or until they mated. If mating did not occur within $10 \mathrm{~min}$, a new virgin female was placed in the Petri dish and the previous female discarded. Dams were subsequently transferred to Petri dishes containing approximately 120 black-eyed beans and remained there for the rest of their lives. Sires were transferred to eppendorf tubes and rested there for $24 \mathrm{~h}$ until they were placed in a new $30-\mathrm{mm}$ petri dish together with a new virgin female (dam). This process was repeated after another $24 \mathrm{~h}$, so that all sires mated with three dams over a period of 3 days. In addition, a subsample of sires $(n=46)$ were weighed before and after their first mating to estimate the size of their first ejaculate (this measure is repeatable-weights before and after copulation were measured two times for a small beetle sample $(N=9)$ and weight loss (= ejaculate size) was calculated two times from these. Regression of measure one on measure two indicates a very high repeatability: $R^{2}=0.967 ; \beta=1.08 ; P<0.0001$ ), and for 60 sires we also measured their body size (mass) and copulation duration. In total, 101 males were mated to 303 females, but after excluding females that did not lay eggs and families with less than two male offspring, data were obtained from a total of 282 full-sib families (100 sires) and 918 sons. Subsequent sample sizes vary somewhat because of lost data or failure of all sons to mate as required (for example).

Virgin sons emerging from these families were isolated as above (from beans with only one egg attached) and the date of emergence was recorded. The first three sons to emerge from each family were subsequently placed with an unrelated virgin female (also collected from the sire-dam matings above) until they mated (the use of daughters in these matings also enabled us to estimate the heritability of copulation duration through female offspring). As before, matings were staged in Petri dishes with $30 \mathrm{~mm}$ diameter, but with each son mated to only one female, and as above, a sample of sons were weighed before and after copulation to estimate ejaculate size and subsequently, its heritability. We chose sons from sires in the top and bottom $10 \%$ of the sire ejaculate size distribution as this minimises the sample sizes required for sire-son (parent-offspring) regression, while maintaining experimental power (Hill, 1970). We thus obtained ejaculate size for 18 sires and 46 sons. Once paired, the beetles were observed continuously until they finished mating. Courting males approach females from behind and antennate females' backs while attempting to mount. Once males successfully insert their aedeagus, they cease waving their antennae and lean back, remaining motionless (Savalli and Fox, 1998). This shift in posture and behaviour was scored as initiation of mating. The point at which sons and their mates physically separated was taken as the end of copulation. For each mating, we estimated copulation duration as the time between copulation start and male-female separation. Sons were then placed in individual eppendorf tubes and checked daily until death. As we knew their age at mating, this allowed us to calculate the total longevity of sons and their longevity after copulation.

\section{Statistical analyses}

Copulation duration, longevity after copulation and age at copulation were transformed ( $\log _{10}$ or Box-Cox) to improve normality and residuals in all analyses were normal $(P>0.6)$ and there were no associations between predicted and residual values. However, estimation of the genetic parameters was virtually identical when transformed or untransformed data were used in our analyses, so estimates from the untransformed data are 
presented here-their interpretation is easier (see Falconer, 1989). The phenotypic association between copulation duration and subsequent longevity was assessed using linear regression. Analyses of genetic variation were conducted on sire and dam variance components estimated with Restricted Maximum Likelihood (SPSS). Significance of the sire estimates was tested with G-tests. For ejaculate size, sire-son regression (following recommendations of Hill, 1970) was employed. Sire estimates of heritability are our focus because dam estimates are confounded by common environment and dominance effects (Lynch and Walsh, 1998; Roff, 2008). Coefficients of additive-genetic variation were also calculated, according to Houle (1992), because they may be a more appropriate measure for comparing potential responses to selection (Price and Schluter, 1991). These coefficients of additive variation were calculated using untransformed values as recommended (Houle, 1992). The genetic correlation between copulation duration and subsequent son's survival were estimated following Becker (1985), using the sums of cross products from a nested MANOVA (SPSS). However, the ReML heritability estimates and s.e. were used in the calculation of $r G$ rather than those derived from the MANOVA because of the unbalanced (final) design (ReML is recommended for unbalanced designs-Lynch and Walsh, 1998). Only this genetic correlation was estimated as potential costs of reproduction were the focus of this article. We also used sire family means to further assess the association between copulation duration and male longevity as family level correlations also provide an estimate of genetic correlations (Lynch and Walsh, 1998).

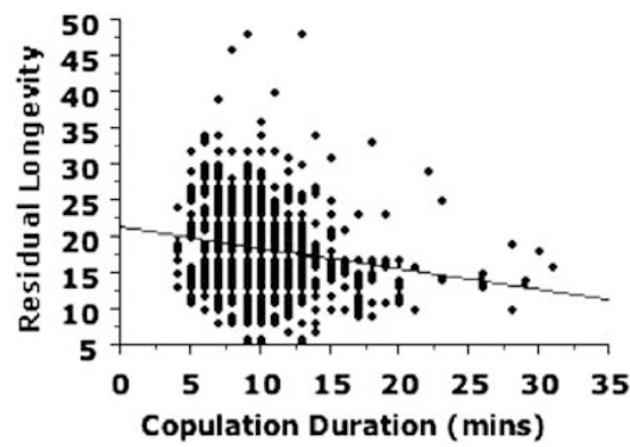

Figure 1 The negative phenotypic association between copulation duration ( $x$ axis) and male longevity (in days after copulation (that is, controlling for age at copulation hence residual)) ( $y$ axis). Shown here are the untransformed data. This relationship is also negative without controlling for male age at the time of copulation and removing the copulations longer than $25 \mathrm{~min}$ only increases the negative slope.

\section{Results}

There was a significant negative association between copulation duration and subsequent longevity in sons at the phenotypic level (Figure 1). This was true whether copulation duration was the only predictor in our model $\left(r^{2}=0.04 ; \beta=-0.24 ; \mathrm{F}_{1,768}=30.9 ; P<0.001\right)$, or if we included son's age at copulation as an additional predictor variable (Overall model $r^{2}=0.17 ; \mathrm{F}_{2,749}=77.3$; $P<0.001$. Copulation duration, $\beta=-0.13 ; t=-3.23$; $P=0.001$. Age at copulation, $\beta=-0.04 ; t=-11.016$; $P<0.001)$. Therefore, at the phenotypic level it appears that males that copulate for longer have reduced longevity. However, with a much reduced sample size, there was no phenotypic association between copulation duration and ejaculate size (body weight lost during copulation) $\left(\mathrm{F}_{1,44}=1.1 ; P=0.3\right)$, nor was there an association between male size (weight) and copulation duration $\left(\mathrm{F}_{1,58}=1.35 ; P=0.25\right)$.

All traits measured were significantly heritable (Table 1) except for ejaculate size $\left(h^{2}=-0.026 ; F_{1,16}\right.$ $<0.0001 ; P=0.92$ ), and for these significant estimates, $\mathrm{CV}_{\mathrm{A}}$ varied from 51.2 to 117.5 (Table 1), all of which indicates there is significant potential for copulation duration, longevity and residual (after copulation) longevity to evolve. Furthermore, copulation duration was heritable through daughters and sons, and additive variation and heritabilites were comparable in both instances. As with the phenotypic association, there was also a negative correlation between longevity after copulation and copulation duration $(r G=-0.16 \pm 0.2)$, although as with genetic correlations generally, the errors on this estimate were relatively large, even with our substantial sample size. Nevertheless, the sign of this association is informative (Lynch and Walsh, 1998) and it indicates there is a negative genetic association between these characters. This inference is supported by sire family level associations between these two traits, which were also negative $\left(\beta=-0.45 ; \mathrm{F}_{1,97}=6.75 ; P=0.01\right)$.

\section{Discussion}

The duration of males' first copulation was negatively associated with their longevity at both the phenotypic and genetic level, and both these traits had substantial additive-genetic variance. The phenotypic association could be due to general male quality, with poor quality males taking longer to copulate and dying quicker. However, the negative genetic correlation suggests that there is a genuine trade-off between male reproductive effort and longevity, and genotypes that invest more in the duration of their first copulation, die faster. Furthermore, the phenotypic association occurs when we control for male age at mating, so it is not simply that older males mate for longer. We also acknowledge that the s.e.

Table 1 Phenotypic means and genetic estimates for the traits we measured that had significant heritabilities

\begin{tabular}{|c|c|c|c|c|c|c|}
\hline Trait & $\mathrm{N}$ & Mean \pm s.e. & $V_{A}$ & $C V_{A}$ & $h^{2}$ & $\mathrm{P}$ \\
\hline Longevity (total: days) & 761 & $19.7 \pm 0.2$ & 19.3 & 100.6 & $0.61 \pm 0.17$ & $<0.0001$ \\
\hline Longevity (residual: days) & 771 & $18.4 \pm 0.2$ & 24.4 & 117.5 & $0.66 \pm 0.18$ & $<0.0001$ \\
\hline Copulation duration (sons: minutes) & 850 & $9.9 \pm 0.1$ & 4.96 & 60.3 & $0.36 \pm 0.14$ & 0.0052 \\
\hline Copulation duration (daughters: minutes) & 838 & $9.9 \pm 0.1$ & 3.56 & 51.2 & $0.26 \pm 0.12$ & 0.02 \\
\hline
\end{tabular}

$N$, phenotypic samples size, mean is the phenotypic mean ( \pm the s.e.), additive genetic variance $\left(\mathrm{V}_{\mathrm{A}}\right)$ and its coefficient of variation $\left(C \mathrm{~V}_{\mathrm{A}}\right)$, sire (narrow-sense) heritability $\left(\mathrm{h}^{2}\right)$ and $P$-value for the heritability estimate. 
of our genetic correlation are relatively large, but this is expected even with this sample size, and it is the sign of the association that is of primary interest to us (Lynch and Walsh, 1998). In addition, correlations at the family level approximate genetic correlations (Lynch and Walsh, 1998), and hence the congruence between these two analyses supports the notion that copulation duration and longevity are negatively genetically correlated. In total then, we provide evidence for a phenotypic tradeoff, and for a genetic trade-off. The former defines the fitness surface and selection, the latter, together with significant trait heritability, facilitates a corresponding evolutionary response to any selection because the sign of the two associations are the same-all else being equal (Roff, 2002). These findings are therefore consistent with a standard assumption of life-history models-investment in reproduction trades-off with longevity (Roff, 2002)-but if and how this trade-off varies across environments (for example, Czesak and Fox, 2003) remains to be investigated. In addition, how further copulations influence this trade-off also needs to be assessed, but male investment is greatest in their first copulation (Savalli and Fox, 1999), which is why we focused on this here.

Previous phenotypic findings in this species suggested that mating has a negative effect on male survival (Paukku and Kotiaho, 2005). Our findings are consistent with this and additionally indicate that the duration of a males first copulation, rather than ejaculate size, is linked to the reduction in longevity at both the phenotypic and genetic level. In apparent contrast with our findings, previous work also indicates that larger males could copulate for longer (Paukku and Kotiaho, 2005) and live for longer. However, the previously reported effect of size on longevity was marginal, moderate $(r=0.16)$ and inconsistent across experiments (Paukku and Kotiaho, 2005). As a result we did not measure size for all our beetles and furthermore we were interested in the fundamental genetic correlations, not in how phenotypic trade-offs may be mediated, whether this is via size or via a combination of phenotypic traits (as seems likely). However, we found no significant association between male size and copulation duration at the phenotypic level, which tentatively suggests the survival cost to longer copulations is not solely mediated by male size. Overall, the work on C. maculatus indicates that there are substantial costs of mating for males, as reported for other taxa (for example, Partridge and Farquhar, 1981; Clutton-Brock and Langley, 1997; Hunt et al., 2002; Martin and Hosken, 2004), and for female C. maculatus (Tatar et al., 1993; Messina and Fry, 2003). Part of this substantial effect of copulation duration on male longevity may be due to the fact that in our population males do not feed or drink as adults, but provide females with a substantial ejaculate that can be up to $10 \%$ of body mass (Paukku and Kotiaho, 2005). This means that males are losing both energy and water in their ejaculate, as suggested by the dramatic decline in ejaculate size with successive matings (Savalli and Fox, 1999). It is unclear however, if males that copulate for longer transfer larger ejaculates to females. The limited data we have on this suggests not, and similarly, Savalli and Fox (1998) also find no evidence that copulation duration is associated with ejaculate size (phenotypically or genetically). However, Edvardsson and Canal (2006) manipulated copula- tion duration experimentally showed that longer copulations resulted in the transfer of heavier ejaculates. Nonetheless, the proximate mechanism for our phenotypic result is not immediately obvious, but the associations we detected are frequently only seen under stress (Roff, 2002), as here (where no water or nutrients were supplied to the adults). In addition, ejaculate mass is only one measure of important/costly products that males transfer to females so mass-cost associations are possibly simplistic. Another potential mechanistic explanation for the copulation duration-longevity association is that traits, which give males more control over copulation duration are costly and result in reduced longevity because of this, although this needs to be substantiated. Regardless of the mechanism however, the negative genetic association between copulation duration and longevity shows that genotypes that copulate for longer in their first copulation, live shorter lives. Whether this association varies across environments and populations remains to be established.

Genetic trade-offs between reproductive effort and longevity have been reported in a range of taxa (Gasser et al., 2000; Zwaan et al., 1995; see Prasad and Joshi, 2003 for a discussion in Drosophila melanogaster). Hunt et al., (2006) used artificial selection on male longevity to assess potential trade-offs with reproductive effort in a cricket. They found that in lines selected for decreased longevity, males began sexual signalling at a younger age, signalled more per night and more over their shorter life-spans than males from lines selected for increased longevity. These results were interpreted, among other things, as evidence for the costliness of sexual selection. Our results suggest that copulation itself can be costly, as suggested for other traits like ejaculate and sperm size (Dewsbury, 1982; Pitnick et al., 1995; Hosken, 2001). Other examples of genetic trade-offs have usually focused on females, but as with Hunt et al. (2006) and our study, published work typically finds negative genetic correlations between reproductive effort (for example, fecundity) and longevity (for example, Zwaan et al., 1995; Miyatake, 1997).

All the traits we measured were significantly heritable, except ejaculate size. This may have been due to the smaller sample size for the ejaculate size sire-son regression (but see Hill, 1970), although Savalli and Fox (1998) also found no significant sire component to ejaculate size variation. However, they reported a significant dam estimate, but because dam additive variances are contaminated with dominance and environmental variance, it is unclear how to interpret this finding. In addition, Savalli and Fox (1998) reported copulation duration heritability estimates that were very close to ours ( 0.25 and 0.35 vs 0.26 and 0.36 ), although unlike here they only found an effect via daughters not sons. They interpreted this as evidence for female determination of copulation duration, but our data suggest this is not the case in our population and that males and females determine how long copulation lasts. The difference between these two studies is not surprising as the animals used have different founders (gene frequencies partly determine heritabilities; Falconer, 1989), and estimates of inheritance patterns of seedbeetle ejaculate size also apparently differ across populations (Savalli and Fox, 1998; Savalli et al., 2000). Furthermore, even studies replicated across labs with the same animals can generate very different outcomes 
(Ackermann et al., 2001), consistent with theory (Falconer, 1989). However, the fact that copulation duration was significantly heritable through daughters and sons does not change our interpretation of the male copulation duration-longevity trade-off-sons were mated to a random assortment of females and this could not generate the negative genetic association within sons or across families. The heritability of copulation duration was also similar to that found in other taxa. For example, Gromko et al. (1991) also reported realised heritabilities of around 23\% and Mühlhäuser et al. (1996) reported a value of 0.39 in yellow dung flies. In the dung fly, copulation duration is an important fitness component because it determines the quantity of rival male sperm displaced, and hence a male's paternity share (Simmons et al., 1996). Rival sperm also appear to be displaced using self-sperm in C. maculatus (Eady, 1994), so copulation duration is also likely to be an important male fitness component here, even if the link between copulation duration, ejaculate size and paternity remains unclear.

In a review of trait heritability, Mousseau and Roff (1987) reported mean values for a range of characters. They found that on average, behavioural traits tended to have higher heritability than life-history characters, and interpreted this result in terms of selection eroding additive-genetic variation in characters more closely related to fitness. We find the opposite pattern here as copulation duration was less heritable than either measure of longevity, and more recently Roff (2002) reported smaller non-significant differences between character classes that were significantly different in the older analysis. Hoffmann (1999) has argued that comparing the heritabilities of behavioural to other trait classes is probably meaningless because low repeatabilities of single-event measures rather than a history of directional selection may confound comparisons. Previous work on C. maculatus also found a genetic component and maternal effects influencing longevity, and evidence for an additive-genetic component to male longevity (Fox et al., 2004), as we find here. However, the relatively high values for measures of male longevity that we report may reflect longevities relatively weak association with fitness in our stocks with our husbandry techniquesthere is no advantage to living an extremely long time in our stocks-although there is clearly selection on longevity as animals must survive long enough to find a mate.

In conclusion, we find evidence for a trade-off between an aspect of male reproductive effort (the duration of a male's first copulation) and longevity. Thus males may be similar to females in this regard. How this varies across environments and male ages remains to be determined. Nevertheless, results are generally consistent with life-history theory and indicate that copulation duration, like other sexual characters, is costly.

\section{Acknowledgements}

This project was funded by NERC and the Leverhulme Trust. T Tregenza is supported by the Royal Society. We thank John Hunt for discussion, and Takahisa Miyatake, Kensuke Okada and two anonymous referees who kindly commented on previous versions of this article.

\section{References}

Ackermann M, Bijlsma R, James AC, Partridge L, Zwaan BJ, Stearns SC (2001). Effects of assay conditions in life history experiments with Drosophila melanogaster. J Evol Biol 14: 199-209.

Arnqvist G, Rowe L (2005). Sexual Conflict. Princeton University Press: Princeton.

Bailey WJ, Withers PC, Endersby M, Gaull K (1993). The energetic costs of calling in the bushcricket Requena verticalis (Orthoptera: Tettigoniidae: Listroscelidinae). J exp Biol 178: 21-37.

Becker WA (1985). Manual of Quantitative Genetics. Washington State University: Pullman.

Blanckenhorn WU, Hosken DJ, Martin OY, Reim C, Teuschl Y, Ward PI (2002). The costs of mating in the dung fly Sepsis cynipsea. I. Costs of copulation. Behav Ecol 13: 353-358.

Burk T (1982). Evolutionary significance of predation on sexually signalling males. Fla Entomol 65: 90-104.

Chapman T, Liddle LF, Kalb JM, Wolfner MF, Partridge L (1995). Costs of mating in Drosophila melanogaster females is mediated by male accessory gland products. Nature 373: 241-244.

Clutton-Brock T, Langley P (1997). Persistent courtship reduces male and female longevity in captive tsetse flies Glossina morsitans morsitans Westwood (Diptera: Glossinidae). Behav Ecol 8: 392-395.

Crudgington HS, Siva-Jothy MT (2000). Genital damage, kicking and early death. Nature 407: 655-656.

Czesak ME, Fox CW (2003). Evolutionary ecology of egg size and number in a seed beetle: genetic trade-off differs between environments. Evolution 57: 1121-1132.

Dewsbury DA (1982). Ejaculate costs and male choice. Am Nat 119: 601-610.

Eady PE (1991). Sperm competition in Callososbruchus maculatus. PhD Dissertation. University of Sheffield. Sheffield.

Eady P (1994). Sperm transfer and storage in relation to sperm competition in Callosobruchus maculatus. Behav Ecol Sociobiol 35: 123-129.

Eady PE, Hamilton L, Lyons RE (2007). Copulation, genital damage and early death in Callosobruchus maculatus. Proc $R$ Soc Lond B 274: 247-252.

Edvardsson M, Canal D (2006). The effects of copulation duration in the bruchid beetle Callosobruchus maculatus. Behav Ecol 17: 430-434.

Edvardsson M, Tregenza T (2005). Why do male Callosobruchus maculatus harm their mates? Behav Ecol 16: 788-793.

Falconer DS (1989). Introduction to Quantitative Genetics, 3rd edn. John Wiley and Sons: New York.

Fox CW, Bush ML, Roff DA, Wallin WG (2004). Evolutionary genetics of lifespan and mortality rates in two populations of the seed beetle, Callosobruchus maculatus. Heredity 92: $170-181$.

Gage MJG (1991). Risk of sperm competition directly affects ejaculate size in the Mediterranean fruit fly. Anim Behav 42: 1036-1037.

Gage MJG, Baker RR (1991). Ejaculate size varies with sociosexual situation in an insect. Ecol Entomol 16: 331-337.

Gasser M, Kaiser M, Berrigan D, Stearns SC (2000). Life-history correlates of evolution under high and low adult mortality. Evolution 54: 1260-1272.

Gromko MH, Briot A, Jensen SC, Fukui HH (1991). Selection on copulation duration in Drosophila melanogaster: predictability of direct versus unpredictability of correlated responses. Evolution 45: 69-81.

Hill WG (1970). Design of experiments to estimate heritability by regression of offspring on selected parents. Biometrics $\mathbf{2 6}$ : 566-571.

Hodgson DJ, Hosken DJ (2006). Sperm competition promotes the exploitation of rival ejaculates. J Theor Biol 243: 230-235. 
Hoffmann AA (1999). Is the heritability for courtship and mating speed in Drosophila low? Heredity 82: 158-162.

Horng S-B (1997). Larval competition and egg-laying decisions by the bean weevil, Callosobruchus maculatus. Anim Behav 53: $1-12$

Hosken DJ (2001). Sex and death: microevolutionary trade-offs between reproductive and immune investment in dung flies. Curr Biol 11: R379-R380.

Hosken DJ, Bailey WJ, O'Shea JE, Roberts JD (1994). Localization of insect calls by the bat Nyctophilus geoffroyi (Chiroptera: Vespertilionidae): a laboratory study. Aust J Zool 42: 177-184.

Houle D (1992). Comparing evolvability and variability of quantitative traits. Genetics 130: 195-204.

Hunt J, Jennions MD, Spyrou N, Brooks R (2006). Artificial selection on male longevity influences age-dependent reproductive effort in the black field cricket Teleogryllus commodus. Am Nat 168: E72-E86.

Hunt J, Simmons LW, Kotiaho JS (2002). A cost of maternal care in the dung beetle Onthophagus taurus. I Evol Biol 15: 57-64.

Kenagy GJ, Trombulak SC (1986). Size and function of mammalian testes in relation to body size. J Mamm 67: 1-22.

Kotiaho JS, Alatalo RV, Mappes J, Nielsen MG, Parri S, Rivero A (1998). Energetic costs of size and sexual signalling in a wolf spider. Proc R Soc Lond B 265: 2203-2209.

Lynch M, Walsh B (1998). Genetics and Analysis of Quantitative Traits. Sinauer: Sunderland, MA.

MacBean IT, Parson PA (1967). Directional selection for duration of copulation in Drosophila melanogaster. Genetics 56: 233-239.

Martin OY, Hosken DJ (2002). Strategic ejaculation in the common dung fly Sepsis cynipsea. Anim Behav 63: 541-546.

Martin OY, Hosken DJ (2004). Copulation reduces male but not female longevity in Saltella sphondylli (Diptera: Sepsidae). J Evol Biol 17: 357-362.

Messina FJ, Fry JD (2003). Evironment-dependent reversal of a life history trade-off in the seed beetle Callosobruchus maculatus. I Evol Biol 16: 501-509.

Miyatake T (1997). Genetic trade-off between early fecundity and longevity in Bactrocerca cucurbitae (Diptera: Tephritidae). Heredity 78: 93-100.

Mousseau TA, Roff DA (1987). Natural selection and the heritability of fitness components. Heredity 59: 181-197.

Mühlhäuser C, Blanckenhorn WU, Ward PI (1996). The genetic component of copulation duration in the yellow dung fly. Anim Behav 51: 1401-1407.

Partridge L, Farquhar M (1981). Sexual activity reduces lifespan of male fruitflies. Nature 294: 580-582.

Paukku S, Kotiaho JS (2005). Costs of reproduction in Callosobruchus maculatus: effects of mating on male longevity and the effects of male mating status on female longevity. J Insect Physiol 51: 1220-1226.

Pitnick S, Markow TA, Spicer GS (1995). Delayed male maturity is a cost of producing large sperm in Drosophila. Proc Natl Acad Sci USA 92: 10612-10618.

Prasad NG, Joshi A (2003). What have two decades of laboratory life-history evolution studies on Drosophila melanogaster taught us? J Genet 82: 45-76.
Price T, Schluter D (1991). On the low heritability of life-history traits. Evolution 45: 853-861.

Qi Y, Burkholder WE (1982). Sex pheromone biology and behaviour of the cowpea weevil Callosobruchus maculatus (Coleoptera: Bruchidae). J Chem Ecol 8: 527-534.

Reznick D (1985). Costs of reproduction: an evaluation of the empirical evidence. Oikos 44: 257-267.

Roff DA (2008). Comparing sire and dam estimates of heritability: jacknife and likelihood approaches. Heredity 100: $32-38$

Roff DA (2002). Life History Evolution. Sinauer: Sunderland, MA. Rose MR, Charlesworth B (1981). Genetics of life history in Drosophila melanogaster. I. Sib analysis of adult females. Genetics 97: 173-186.

Savalli UM, Fox CW (1999). The effect of male mating history on paternal investment, fecundity and female remating in the seed beetle Callosobruchus maculatus. Funct Ecol 13: 169-177.

Savalli UM, Fox CW (1998). Genetic variation in paternal investment in a seed beetle. Anim Behav 56: 953-961.

Savalli UM, Czesak ME, Fox CW (2000). Paternal investment in the seed beetle Callosobruchus maculatus (Coleoptera: Bruchidae): variation among populations. Ann Entomol Soc Am 93: 1173-1178.

Schwarzenbach GA, Hosken DJ, Ward PI (2005). Sex and immunity in the yellow dung fly Scathophaga stercoraria. J Evol Biol 18: 455-463.

Simmons LW, Stockely P, Jackson RL, Parker GA (1996). Sperm competition or sperm selection: no evidence for female influence over paternity in yellow dung flies Scatophaga stercoraria. Behav Ecol Sociobiol 38: 199-206.

Simmons LW, Craig M, Llorens T, Schinzig M, Hosken D (1993). Bushcricket spermatophores vary in accord with sperm competition and parental investment theory. Proc $R$ Soc Lond B 251: 183-186.

Siva-Jothy MT, Tsubaki Y, Hooper RE (1998). Decreased immune response as a proximate cost of copulation in a damselfly. Physiol Entomol 23: 274-277.

Tatar M, Carey JR, Vaupel JW (1993). Long-term cost of reproduction with and without accelerated senescence in Callosobruchus maculatus-Analysis of age-specific mortality. Evolution 47: 1302-1312.

Thornhill R, Alcock J (1983). The Evolution of Insect Mating Systems. Harvard University Press: Harvard.

Tuttle MD, Ryan MJ (1981). Bat predation and the evolution of frog vocalizations in the neotropics. Science 214: 677-678.

Van Voorhies WA (1992). Production of sperm reduces nematode lifespan. Nature 360: 456-458.

Wedell N, Gage MJG, Parker GA (2002). Sperm competition, male prudence and sperm-limited females. Trends Ecol Evol 17: 313-320.

Wigby S, Chapman T (2005). Sex peptide causes mating costs in females. Curr Biol 15: 316-321.

Zwaan B, Bijlsma R, Hoekstra RE (1995). Direct selection on life-span in Drosophila melanogaster. Evolution 49: 649-659. 